\title{
PIWIMS: Physics Informed Warehouse Inventory Monitory via Synthetic Data Generation
}

\author{
João Falcão \\ Carnegie Mellon University \\ USA \\ joaodf@cmu.edu \\ Akkarit Sangpetch \\ CMKL University \\ Thailand \\ akkarit@cmkl.ac.th
}

\author{
Prabh Simran Baweja \\ Carnegie Mellon University \\ USA \\ prabh@cmu.edu
}

Hae Young Noh

Stanford University

USA

noh@stanford.edu

\author{
Yi Wang \\ Carnegie Mellon University \\ USA \\ yiwang2@andrew.cmu.edu \\ Orathai Sangpetch \\ CMKL University \\ Thailand \\ orathai@cmkl.ac.th
}

\author{
Pei Zhang \\ University of Michigan \\ USA \\ peizhang@umich.edu
}

\begin{abstract}
State-of-the-art camera-based deep learning methods for inventory monitoring tend to fail to generalize across different domains due to the high variance of scene settings. Large amounts of human labor are required to label and parameterize the models, making a real-world deployment impractical. In a third-party warehouse setting, supervised learning approaches are either too costly and/or inaccurate to deploy due to the need for human labor to address the diverse set of environmental factors (i.e, lighting conditions, product motion, deployment limitations).

We introduce PIWIMS, a realistic synthetic dataset generation technique that combines the physical constraints of the scene in real-world deployments, drastically reducing the need for human labeling. In contrast to other generative techniques, where the generative parameters are learned from a large sample of available data, this compositive approach defines the parameters based on physical characteristics of the particular task, which requires minimal human annotation. We demonstrate PIWIMS performance in a 4-month real operating warehouse deployment and show that with only 32 manually labeled images per object, PIWIMS can achieve an accuracy of up to $87 \%$ in inventory tracking, which is a $28 \%$ increase when compared to traditional data augmentation techniques and $31 \%$ error reduction when compared to the third-party warehouse industry average. Furthermore, we demonstrate the ability of PIWIMS to generalize across different camera angles and positions by achieving an accuracy of $85 \%$ in inventory tracking while varying the position and angle of the camera.
\end{abstract}

This work is licensed under a Creative Commons Attribution International 4.0 License.

UbiComp-ISWC '21 Adjunct, September 21-26, 2021, Virtual, USA

(c) 2021 Copyright held by the owner/author(s).

ACM ISBN 978-1-4503-8461-2/21/09.

https://doi.org/10.1145/3460418.3480415

\section{CCS CONCEPTS}

- Computing methodologies $\rightarrow$ Image representations; Physical simulation; Scientific visualization.

\section{KEYWORDS}

Synthetic Dataset, Computer Vision, Real World Deployments, Product Recognition, Inventory Monitoring

\section{ACM Reference Format:}

João Falcão, Prabh Simran Baweja, Yi Wang, Akkarit Sangpetch, Hae Young Noh, Orathai Sangpetch, and Pei Zhang. 2021. PIWIMS: Physics Informed Warehouse Inventory Monitory via Synthetic Data Generation. In Adjunct Proceedings of the 2021 ACM International foint Conference on Pervasive and Ubiquitous Computing and Proceedings of the 2021 ACM International Symposium on Wearable Computers (UbiComp-ISWC '21 Adjunct), September 21-26, 2021, Virtual, USA. ACM, New York, NY, USA, 6 pages. https://doi. org/10.1145/3460418.3480415

\section{INTRODUCTION}

The publication of large-scale object recognition datasets has enabled an unprecedented use of camera-based systems using deep learning. While supervised learning approaches have shown success in classification, detection, and segmentation, these, however, fail to generalize well in the absence of large-scale labeled datasets [18]. Particularly in inventory monitoring applications where a large number of specific objects - e.g. products in retail store, products flowing in and out of a warehouse; and a vast diversity of task-specific environmental factors are present (see Figure 1). Such applications require immense human labor to label data and parameterize the model, making it impractical for real-world adoption.

In order to address this issue, recent techniques, such as domain adaptation techniques [25], data augmentation [19], incremental learning [27], few-shot learning [14] and automatically labeling techniques [17], have appeared to reduce the amount of human labor required in order to adapt the learned models to the domain and data distribution of the specific application. Synthetic data generation aims at creating a large dataset out of a very small 


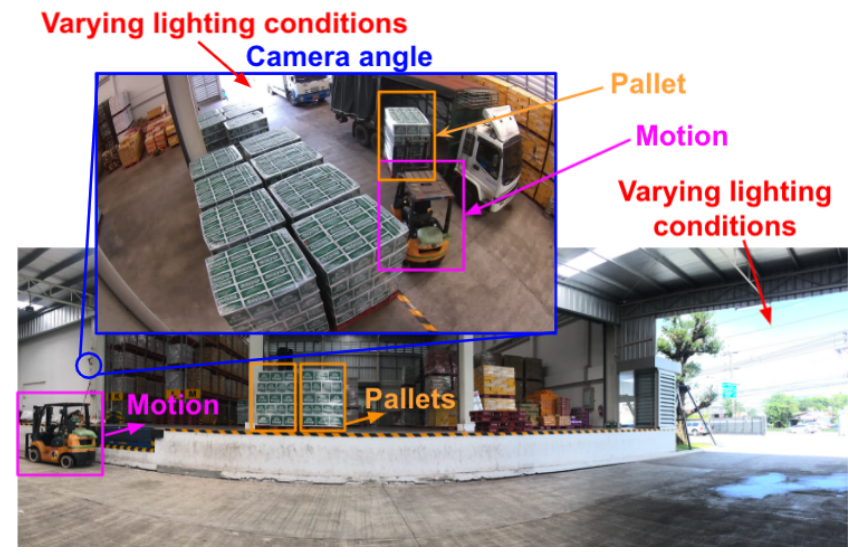

Figure 1: Typical warehouse setup with forklifts moving the pallets in and out. Diverse set of environmental factors are observed: motion specific to forklifts, camera angle limitation, varying lighting conditions impacted by the weather.

amount of labels by varying characteristics of the original labeled sample. While this allows the trained model to become robust to such variances, it will further over-fit to the patterns in the augmentation itself.

In this work, we present PIWIMS which explores different ways of generating synthetic warehouse datasets by leveraging physical knowledge of the deployment setup and object through compositing augmentation to bind the generative parameters closer to the physical reality with minimal human annotation. Our contributions are as follows:

(1) We propose a new generative data-augmentation technique PIWIMS, which leverages physical information -scene lighting, camera position and angle, and object motion, size, and shape- to generate datasets that more closely resemble the deployment data distributions with minimum human annotation labor.

(2) We present an analysis of a 4 month deployment of PIWIMS used in a real world warehouse inventory monitoring system.

(3) We demonstrate the ability of PIWIMS to generalize across camera deployments with varying position and angle.

The remaining paper is as follows: Section 2 describes in detail our approach. Section 3 presents the experimental results and analysis of PIWIMS in real-world warehouse setting. Section 4 shows previous works on data augmentation techniques and learning with limited labeled data. Finally, we conclude in Section 5.

\section{PIWIMS OVERVIEW}

PIWIMS takes inspiration from the ability humans have to look at an image and project different placements for objects of interest. To project an object to a different place in an image, humans have to have a rough understanding of the physical layout of the scene (where the floor is, natural orientation of the object, sources of light, natural motion). This rough understanding of the physical context of a scene is designed into our approach.
Like other compositing techniques [7, 22], PIWIMS requires as input a background image, $b$, and a target image crop, $c$. Our approach requires as well a linear transformation function, $T$, and a color correction function, $M$, and finally applies an alpha blending technique [10] to compose the positive training case. The approach focuses on constraining the parameters of $T$ and $M$ closer to the bounds of physical possibility, while still exploring a larger data distribution. In the simplest case, the final training image is then computed by: $i=b \oplus M(T(c))$ (where " $\oplus$ " corresponds to the alpha blending). However, we can consider multiple $c$ 's and end up with the following formulation:

$$
\begin{aligned}
& i=b \oplus M_{x_{1}}\left(T_{y_{1}}\left(c_{1}\right)\right) \oplus M_{x_{2}}\left(T_{y_{2}}\left(c_{2}\right)\right) \\
& \ldots \oplus M_{x_{n}}\left(T_{y_{n}}\left(c_{n}\right)\right), \quad \forall x \in \mathcal{M}, \forall y \in \mathcal{T}
\end{aligned}
$$

Note that each $M_{x}$ and $T_{y}$ are different color correction and linear transformations respectively.

\subsection{Physically informed linear transformation}

The linear transformation defined by $T$ in Eq. 1 is further defined by the input parameters $(X, \theta, s)$. $X$ represents the final location of the center of mass of the cropped image $(c)$ in $\mathcal{R}^{2} . X$ can vary randomly to any point in the image. $\Theta$ however is constrained by the maximum rotation that $c$ can see given the application-specific setup. This is computed manually by placing $c$ in its original background $b$ at the highest/lowest rotation possible. By manually placing the crop $c$, PIWIMS is bounding the space of possible rotations for $c$. We define the possible set of rotations as $\mathcal{Y}$.

Similarly, the size $S$ of the crop $c$ is equally computed by manually placing $c$ in the original background $b$. In this case, the annotator will resize the crop to the largest and smallest possible perceived size given the application-specific setup. Once again, bounding the possible sizes of $c$ to the physically reasonable ones. The size of $c$ is defined by the ratio of the area (number of pixels) of the crop $c$ and the area of $b$, and its possible space is defined as $\mathcal{S}$. Finally $T \in \mathcal{T}$ where $\mathcal{T}=(\boldsymbol{Y}, \mathcal{S})$.

\subsection{Physically informed color correction}

When bounding the possible synthetic images in the color space, we focused on lighting. This is formulated through the function $M$ present in Eq. 1. $M$ represents the color space of the $c$ image in HSL (Hue, Saturation and Lightness) space. In order to encode the lighting characteristics (origin, color, and intensity) easily intuited by humans, PIWIMS requires the annotator to understand the lighting in the application-specific setup and place the cropped image $c$ in the original background $b$, and, similarly to subsection 2.1 , search for the maximum/minimum hue and lightness value possible. The range computed between the maximum and minimum values define the space $\mathcal{M}$. Our approach ignores saturation based on the fact that $c$ is extracted from the same source that the model will operate in, which already includes the data characteristics of the light sensor. This assumption can further be alleviated to improve the model.

While this is a homogeneous color mapping transformation, we believe that it is possible to enhance this model with a directed lighting source. 


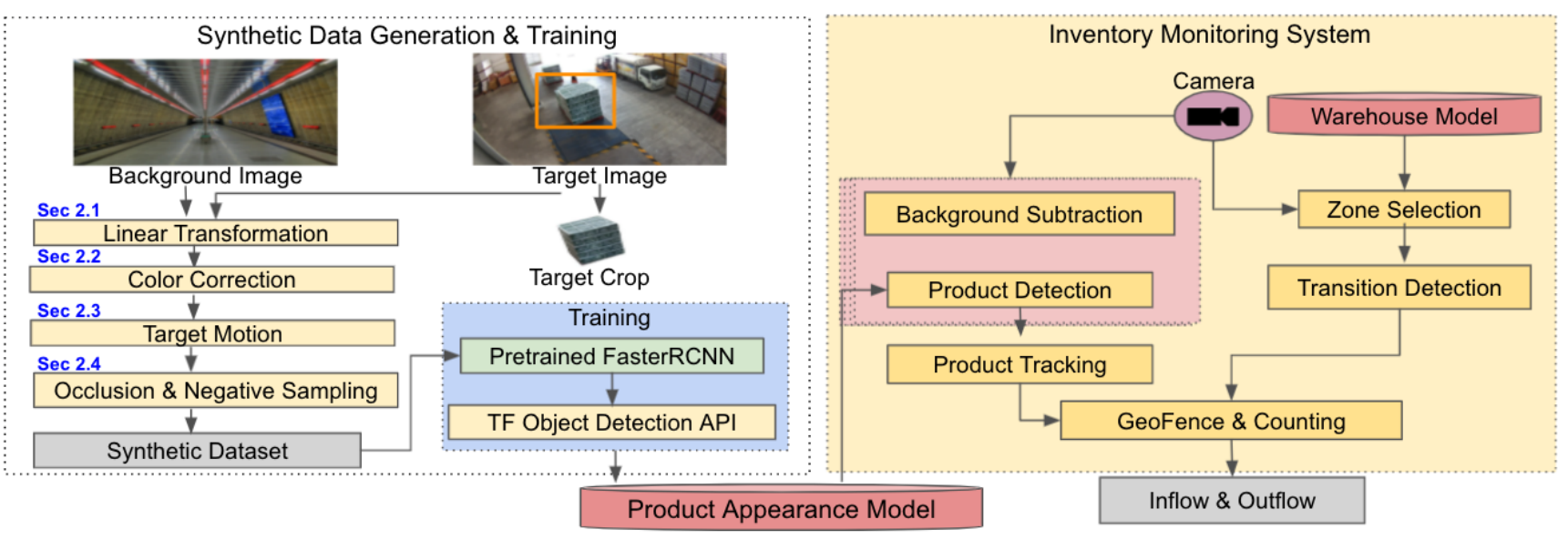

Figure 2: PIWIMS consists of two steps: randomly sampling a background image and a target crop, selecting the PIWIMS approach, and generating the dataset according to the desired physical characteristics of the scene and the object. The generated dataset is used to train the product appearance model that is fed to the Inventory Monitoring System.

\subsection{Target Motion}

Even though the motion is associated with a continuous set of images (video), it can still be perceived in a static image. Motion blur can be detected in a single image, indicating a natural motion that creates a loss of detail due to the capture rate of the camera's sensor. The same principles applied in Section 2.1 and Section 2.2 can be applied here. Motion blur can be applied by a convolution between a kernel $k$, defining the direction and intention of the motion, and the image $c$.

In cases where the ordered series of images is available, motion can further be used through a pre-processing step of background subtraction [20]. PIWIMS transforms the background image $b$ using a synthetic background subtraction removal function $f . f$ randomly samples the coordinate space of $b$ and converts that pixel into a black pixel with probability $p$. In case one of its 8 neighboring pixels has been converted to black its "turning black" probability doubles becoming $(1+p) / 2$. This is defined as such:

$$
P\left(b_{x, y}=0 \mid p\right)=\left\{\begin{array}{cl}
p & , \forall x, y \text { where } b_{|x-1|,|y-1|} \neq 0 \\
\frac{(1+p)}{2} & , \forall x, y \text { where } b_{|x-1|,|y-1|}=0
\end{array}\right.
$$

We have identified empirically that $p=0.53$ has the best performance when reproducing the effects of background subtraction. See 2c). for an example of a resulting background images with $f(b, 0.53)$.

\subsection{Occlusion and Negative Sampling}

When blending the background, $b$, with the multiple target cropped images, c's, [22] has demonstrated that the artifacts created by composing the final image can be identified by the training model, which leads to it expecting such artifacts in the application-specific images. Therefore PIWIMS further adds similarly shaped, to $c$, extra cropped images denoted as $v$ with content from the different random background, in order to create negative samples with the compositing artifacts. (See Fig. 3). The last step of PIWIMS is to remove samples generated with extreme occlusions by applying
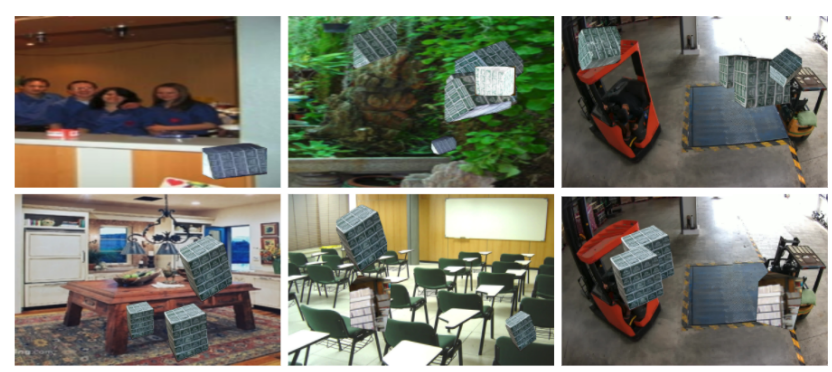

Figure 3: The top three images show the effect of random dataset generation that lies outside the physical reality of the application. The bottom three images demonstrate the generation process based on PIWIMS linear transformation, color correction and negative sampling.

NMS (non-maximum suppression) with a threshold of 0.8 on the generated $c$ images.

\section{REAL-WORLD EVALUATION AT AN OPERATING WAREHOUSE}

In this section, we present a component-wise analysis of the different stages of PIWIMS and the results of the deployment of PIWIMS in a real-world operating warehouse monitoring system. In partnership with ThaiBev, we have deployed a warehouse inventory monitoring system based on the data generated by PIWIMS. We analyze in this paper 4-month of operation of this warehouse. And further evaluate each component of PIWIMS in Sections 2.1, 2.2 and 2.3 (See Fig. 2).

\subsection{Warehouse camera deployment and dataset generation}

We deployed two cameras at a ThaiBev's warehouse for varying camera positions and angles. The cameras' placement is chosen in 


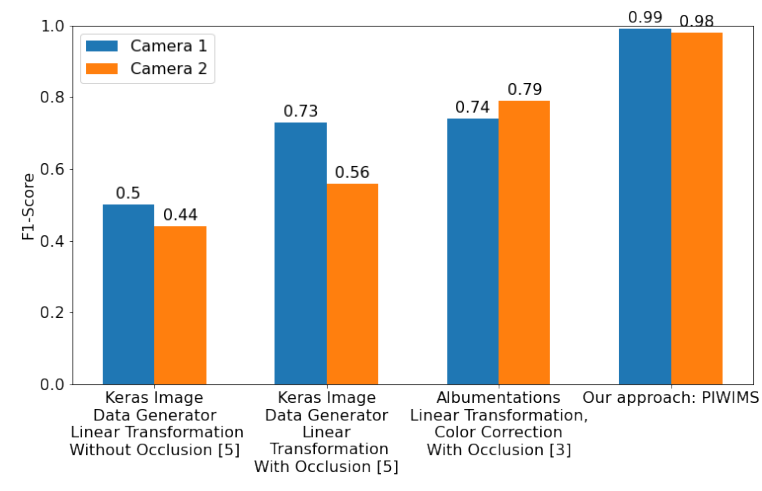

Figure 4: Detection Accuracy results with $\mathrm{IoU}=0.8$, using fully synthetic datasets generated using various data augmentation techniques. We show that PIWIMS with minimum human labor (only 32 manually labeled images) outperforms the other techniques for both the deployments.

a way to capture the maximum activity occurring at each door. The cameras operated at $15 \mathrm{fps}$ and recorded all activities during the four months of the deployment. The deployment was coordinated to record the 4 busiest months of the year. We have further obtained the inventory count from a separate warehouse monitoring system (WMS) for comparison.

Ground truth was generated by manually annotating 35 hours of randomly selected video frames from different days and cameras. We further evaluate the effectiveness of our approach by comparing our visual inventory monitoring results with ThaiBev's WMS results.

Dataset generation was achieved by extracting 32 cropped product images of the highest selling item in the warehouse from each deployed camera, $c_{n}$. We have further leveraged the Indoor Scene Recognition Dataset [13] to generate our backgrounds $b$ for an increased variety of backgrounds which facilitates the generalization process. By following the approach describe in Sec. 2, we create fully synthetic datasets of 30,000 images out of the 32 cropped sampled images (with a 20:20:60::validation:testing:training dataset composition), for each component provided in Sec. 2 (See Fig. 2). We then train a FasterRCNN [15] model with the synthetically generated datasets for the different steps in PIWIMS.

\subsection{Metrics for Product detection and Product flow counting}

Inventory Monitoring requires the vision systems to detect the products of interest and track their flow over time. For this purpose, we have divided our analysis of the results into Product detection accuracy and Product flow counting.

Product Detection is a localization and classification task, which we evaluate using the F1-score @ $0.8 \mathrm{IoU}$, that is $\frac{|G \bigcap P|}{|G \bigcup P|}>=0.8$, where $G$ is the ground truth bounding box and $P$ is the predicted bounding box.

Product flow counting aims to estimate the locations of products in a video sequence and yield their individual inflow/outflow count based on the entrance and exit zone in the warehouse, which we evaluate using the $\mathrm{F} 1$ score. If the ground truth states a product

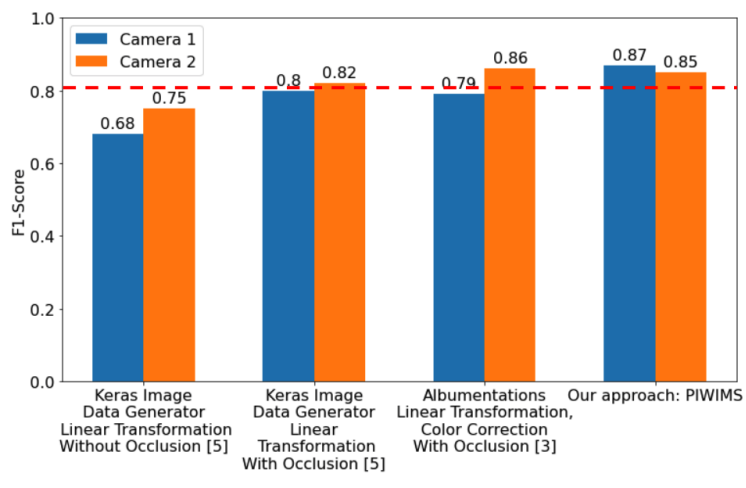

Figure 5: Inventory Count Evaluation Results for different models, using synthetic datasets generated with only 32 manually labeled images. Our approach, PIWIMS has an $8 \%$ performance increase compared to Albumentations [3] 31\% error reduction as compared to WMS (dotted line).

inflow/outflow at time $t_{1}$, and the predicted time states a product inflow/outflow at time $t_{2}$, it is considered a true positive if $\left|t_{1}-t_{2}\right|<=50$, where we consider 50 frames (equivalent to 3.5 seconds) to accommodate for human annotation errors.

\subsection{PIWIMS data augmentation performance}

In order to evaluate the performance of PIWIMS we have leveraged several data augmentation techniques and used them for the warehouse inventory monitoring deployment. We compare our model with Albumentations [3], and various techniques from Keras Image Data Generator [5] on product detection and product flow counting subtasks. The results show that all the components of PIWIMS perform better or on-par with the baseline on respective tasks (See Fig. 4 and 5).

Product Detection: Following the apporach in Sec.2 we create detection models from the synthetically generated datasets for PIWIMS, Albumentations [3] and Keras Image Data Generator [5] methods. We demonstrate in figure 4 that with a minimum amount of human labor (only 32 cropped samples), a combination of all the components of PIWIMS achieves 99\% F1-score @ 0.8 IoU.

Product Flow Counting: We detect the inventory by running our trained models and then use SORT [26] to track the movement in $2 \mathrm{D}$ of the products across time. We further define an entrance and exit zone in the image, determining whether a moving product is considered inflow or outflow. Figure 5 shows that we see an $8 \%$ improvement in F1-score for the first camera and on-par performance for the second camera as compared to other data augmentation techniques.

Furthermore, we compare our model with WMS data (See Figure 5), the 3rd party warehouse industry average. The results demonstrated that our models resulted in a $31 \%$ reduced error as compared to WMS.

\subsection{Generalization across different camera angle and position}

In order to demonstrate the ability of PIWIMS to generalize across different camera installations, we deployed a second camera at the 


\begin{tabular}{|l|c|}
\hline Physically informed parameters & Impacted by \\
\hline \hline Linear Transformations $(\mathcal{Y})$ & Camera angle and position \\
Color Correction $(\mathcal{M})$ & Lighting angle and intensity \\
Motion Blur & Warehouse product motion \\
Occlusion Parameters & Camera position \\
\hline
\end{tabular}

Table 1: Analysis of physical realities that impact the parameters used for data generation in PIWIMS. Notice that, in general, linear transformations and color correction are derived from camera placement motion blur and occlusion parameters are specific to the warehouse characteristics.

same warehouse (see Figure 1). The second camera is deployed at the same height from the warehouse platform as the first camera. As both cameras are deployed in the same warehouse, similar physical information is extracted when generating the synthetic datasets.

In Table 1 we list the factors that impact the physical parameters used in PIWIMS. As the second camera is deployed at the same height as the first camera, the linear transformations $\mathcal{S}$ remain similar across cameras. However, the linear transformations $\mathcal{Y}$ is adjusted according to the angle of the second camera. The color space $\mathcal{M}$ for images collected from the second camera differs from the first camera. This occurs since the first camera is installed close to the door with direct sunlight access, but this is not the case for the second camera installation. Motion blur and occlusion parameters are similar across camera installations as they are specific to the warehouse, and the product of interest moves at similar speeds from both perspectives.

Evaluation was done by running the same trained model produced for the first camera on the second camera. We randomly selected 10 hours of video from the second camera, from different days and times of the day of the four months of the deployment. This data was annotated by manually looking at the video and registering how many inflows and outflows of the desired product occurred during this 10 hours period. Figure 4 and 5 demonstrate that PIWIMS generalizes well across different camera installations without added human labor for annotation. PIWIMS exhibited a $19 \%$ improvement on product detection subtask compared to the other data augmentation techniques and on-par performance on product counting subtask.

\section{RELATED WORK}

There is a significant amount of work in synthetic data generation with the intent of overcoming the burden of labeling large datasets for training neural networks. However, most of these works lack the optimization for the target application and focus on just slightly perturbing the available data distribution. These can be grouped into learning-based image synthesis, photo-realistic mapping and contextual image composition.

Learning-based image synthesis: In order to synthesize positive examples for training object detection models prior works [2, $21,28]$ leverage pre-existing labeled datasets and vary its characteristics (i.e. saturation, brightness, orientation, etc.) to augment the data distribution with the attempt at maintaining the patterns that make such an example a positive one. These parameters can be learned by a model to generate these synthetic images $[4,6]$ or generated through adversarial examples $[1,12,22]$. ST-GAN [10] uses spatial composition to generate new images, however, without the contextual understanding of the target application. These, however, still require a large available labeled dataset to learn meaningful patterns and generate image samples that improve the training performance.

Contextual image composition: Image composition refers to the technique of cropping separate images and composing them in such a way that new images are generated with, ideally, similar data distribution to the target context. While these techniques [7, 8, 24] have shown an increase in performance through the random placement of images crops on random backgrounds, they tend to generate unrealistic datasets, limiting its ability to adapt to new domains [7]. To improve this, data augmentation techniques have adopted a parameterized method to limit the distribution of the composition [2]. However these parameters require a large amount of human labor to fine-tune its performance for each composition. To address this, prior works use heuristical [8] techniques or contextual cues [11] to improve the realism of the composition.

Photo-realistic mapping: Photo-realistic 3D simulation engines, such as game engines, have recently gained popularity for data augmentation purposes $[9,16,23]$. These approaches provide a large degree of control over the possible transformations done to available data and into the realism of the resulting dataset. However, the significant downsides of this approach are the domain gap between simulated images and the real application and the hidden need for human labor to generate and ingest 3D data into the simulator.

\section{CONCLUSION}

We introduce PIWIMS, a physics-informed synthetic data generation technique for real-world deployments. PIWIMS utilizes the physical constraints of the environment and the objects to generate realistic datasets. Our approach requires minimal human annotation, and we demonstrate that with an empirical study of inventory monitoring in a warehouse, where we achieve $87 \%$ accuracy in inventory tracking with only 32 manually labeled images.

While these results show great promise in deploying a vision system with virtually no available data and with minimal human labor, they are still preliminary. Future work will involve evaluating this approach against an increasing variety of products and an increase variety of deployments. In addition, further exploration is required to understand the minimum required number of manually annotated samples are required to perform well.

\section{ACKNOWLEDGMENTS}

This research was supported by Thaibev. We thank all the research members of Thaibev for their help with all the deployments at their warehouse and access to the WMS data. We further thank AiFi, Rongjian Xiao, and Sripong Ariyadech for their support in deploying PIWIMS in an operating warehouse.

\section{REFERENCES}

[1] Sima Behpour, Kris M Kitani, and Brian D Ziebart. 2019. Ada: Adversarial data augmentation for object detection. In 2019 IEEE Winter Conference on Applications of Computer Vision (WACV). IEEE, 1243-1252.

[2] Alex Bewley, Zongyuan Ge, Lionel Ott, Fabio Ramos, and Ben Upcroft. 2016. Simple online and realtime tracking. 2016 IEEE International Conference on Image Processing (ICIP) (Sep 2016). https://doi.org/10.1109/icip.2016.7533003 
[3] Alexander Buslaev, Vladimir I. Iglovikov, Eugene Khvedchenya, Alex Parinov, Mikhail Druzhinin, and Alexandr A. Kalinin. 2020. Albumentations: Fast and Flexible Image Augmentations. Information 11, 2 (2020). https://doi.org/10.3390/ info11020125

[4] Ching-Hang Chen, Ambrish Tyagi, Amit Agrawal, Dylan Drover, Stefan Stojanov, and James M Rehg. 2019. Unsupervised 3d pose estimation with geometric selfsupervision. In Proceedings of the IEEE/CVF Conference on Computer Vision and Pattern Recognition. 5714-5724.

[5] Francois Chollet. 2016. Building powerful image classification models using very little data. Keras Blog (2016).

[6] Ekin D Cubuk, Barret Zoph, Dandelion Mane, Vijay Vasudevan, and Quoc V Le. 2019. Autoaugment: Learning augmentation strategies from data. In Proceedings of the IEEE/CVF Conference on Computer Vision and Pattern Recognition. 113-123.

[7] Nikita Dvornik, Julien Mairal, and Cordelia Schmid. 2018. Modeling visual context is key to augmenting object detection datasets. In Proceedings of the European Conference on Computer Vision (ECCV). 364-380.

[8] Debidatta Dwibedi, Ishan Misra, and Martial Hebert. 2017. Cut, paste and learn Surprisingly easy synthesis for instance detection. In Proceedings of the IEEE International Conference on Computer Vision. 1301-1310.

[9] Stefan Hinterstoisser, Vincent Lepetit, Paul Wohlhart, and Kurt Konolige. 2017 On Pre-Trained Image Features and Synthetic Images for Deep Learning. arXiv:1710.10710 [cs.CV]

[10] Chen-Hsuan Lin, Ersin Yumer, Oliver Wang, Eli Shechtman, and Simon Lucey. 2018. ST-GAN: Spatial Transformer Generative Adversarial Networks for Image Compositing. arXiv:1803.01837 [cs.CV]

[11] Roozbeh Mottaghi, Xianjie Chen, Xiaobai Liu, Nam-Gyu Cho, Seong-Whan Lee, Sanja Fidler, Raquel Urtasun, and Alan Yuille. 2014. The role of context for object detection and semantic segmentation in the wild. In Proceedings of the IEEE Conference on Computer Vision and Pattern Recognition. 891-898.

[12] Xi Peng, Zhiqiang Tang, Fei Yang, Rogerio S Feris, and Dimitris Metaxas. 2018 Jointly optimize data augmentation and network training: Adversarial data augmentation in human pose estimation. In Proceedings of the IEEE Conference on Computer Vision and Pattern Recognition. 2226-2234.

[13] A. Quattoni and A. Torralba. 2009. Recognizing indoor scenes. In 2009 IEEE Conference on Computer Vision and Pattern Recognition. 413-420. https://doi.org/ 10.1109/CVPR.2009.5206537

[14] Sachin Ravi and Hugo Larochelle. 2016. Optimization as a model for few-shot learning. openreview.net (2016)

[15] Shaoqing Ren, Kaiming He, Ross B. Girshick, and Jian Sun. 2015. Faster R-CNN Towards Real-Time Object Detection with Region Proposal Networks. CoRR abs/1506.01497 (2015). arXiv:1506.01497 http://arxiv.org/abs/1506.01497

[16] Stephan R. Richter, Zeeshan Hayder, and Vladlen Koltun. 2017. Playing for Benchmarks. arXiv:1709.07322 [cs.CV]

[17] Carlos Ruiz, Joao Falcao, and Pei Zhang. 2019. AutoTag: Visual Domain Adaptation for Autonomous Retail Stores through Multi-Modal Sensing. In Adjunct Proceedings of the 2019 ACM International foint Conference on Pervasive and Ubiquitous Computing and Proceedings of the 2019 ACM International Symposium on Wearable Computers (London, United Kingdom) (UbiComp/ISWC '19 Adjunct). Association for Computing Machinery, New York, NY, USA, 518-523. https://doi.org/10.1145/3341162.3345604

[18] Jürgen Schmidhuber. 2015. Deep learning in neural networks: An overview. Neural networks 61 (2015), 85-117.

[19] Connor Shorten and Taghi M Khoshgoftaar. 2019. A survey on image data augmentation for deep learning. Journal of Big Data 6, 1 (2019), 1-48.

[20] Andrews Sobral et al. 2013. BGSLibrary: An opencv c++ background subtraction library. In IX Workshop de Visao Computacional, Vol. 27.

[21] Toan Tran, Trung Pham, Gustavo Carneiro, Lyle Palmer, and Ian Reid. 2017. A bayesian data augmentation approach for learning deep models. arXiv preprint arXiv:1710.10564 (2017)

[22] Shashank Tripathi, Siddhartha Chandra, Amit Agrawal, Ambrish Tyagi, James M Rehg, and Visesh Chari. 2019. Learning to generate synthetic data via compositing. In Proceedings of the IEEE/CVF Conference on Computer Vision and Pattern Recognition. 461-470.

[23] Gul Varol, Javier Romero, Xavier Martin, Naureen Mahmood, Michael J Black, Ivan Laptev, and Cordelia Schmid. 2017. Learning from synthetic humans. In Proceedings of the IEEE Conference on Computer Vision and Pattern Recognition. 109-117.

[24] Hao Wang, Qilong Wang, Fan Yang, Weiqi Zhang, and Wangmeng Zuo. 2019 Data augmentation for object detection via progressive and selective instanceswitching. arXiv preprint arXiv:1906.00358 (2019).

[25] Mei Wang and Weihong Deng. 2018. Deep visual domain adaptation: A survey. Neurocomputing 312 (2018), 135-153.

[26] Nicolai Wojke, Alex Bewley, and Dietrich Paulus. 2017. Simple online and realtime tracking with a deep association metric. In 2017 IEEE international conference on image processing (ICIP). IEEE, 3645-3649.

[27] Yue Wu, Yinpeng Chen, Lijuan Wang, Yuancheng Ye, Zicheng Liu, Yandong Guo, and Yun Fu. 2019. Large scale incremental learning. In Proceedings of the IEEE/CVF Conference on Computer Vision and Pattern Recognition. 374-382.
[28] Barret Zoph, Ekin D Cubuk, Golnaz Ghiasi, Tsung-Yi Lin, Jonathon Shlens, and Quoc V Le. 2020. Learning data augmentation strategies for object detection. In European Conference on Computer Vision. Springer, 566-583. 